\title{
Throughput Maximization in Cognitive Radio Networks using Levenberg-Marquardt Algorithm
}

\author{
Pavithra Roy P. ${ }^{1}$, Dr. Muralidhar M. ${ }^{2}$ \\ Asst. Professor, Dept. of Electronics and Communication Engg., Vemana Institute of Technology, Bangalore, India ${ }^{1}$ \\ Principal, Sri Venkateswara College of Engg. \& Technology, Chittoor, India ${ }^{2}$
}

\begin{abstract}
Cognitive radio network is the promising technology of the next generation communication networks which enables the secondary users (SUs) to use the free spectrum bands which are licensed originally to the primary users (PUs) without causing interference and utilize the spectrum more efficiently. Spectrum sensing should be carried out frequently in order to transmit the data successfully through SU without causing significant interference with the PU and to achieve the maximum throughput. In this paper we propose an artificial neural network model which is known as Levenberg-Marquardt (L-M) algorithm for predicting the propagation. The simulation results show that there is a significant gain in the throughput when compared with both random and HMM methods and it explains the superiority of the algorithm. Also, it is found that the L-M algorithm is faster than the other algorithms.
\end{abstract}

Keywords: Cognitive Radio Networks, Throughput, Neural Network, Levenberg-Marquardt algorithm

\section{INTRODUCTION}

Radio frequency spectrum is one of the important limited resources in wireless communications which should be utilized efficiently. It is reported by Federal Communications Commission (FCC) that $70 \%$ of the allocated spectrum bands in US are not fully utilized [1]. Recently, the U.S. Federal Communications Commission [2] has approved the opportunistic use of TV frequency bands for unlicensed users such as WLAN and Wi-Fi. IEEE has announced the IEEE 802.22 wireless network standard [3] that specifies how to utilize the unused resources between channels in the TV frequency spectrum. Cognitive radio networks (CRNs) have emerged as a promising solution for achieving better utilization of the frequency spectrum to satisfy the increasing demand of wireless communication resources. In a CRN, the secondary users are allowed to utilize the frequency bands of primary users when these bands are not currently being used. Spectrum sensing, spectrum decision, spectrum sharing, and spectrum mobility are four major functions of cognitive radio (CR) $[4,5]$ systems. Spectrum sensing is utilized to identify primary user's spectrum occupancy status, based on which CR users can dynamically access the available channels through the regulation processes of spectrum decision, spectrum sharing, and spectrum mobility. To avoid collisions with other CRs, after sensing and probing a channel, the $\mathrm{CR}$ needs to make a decision on whether to terminate the scan and use the underlying channel or to skip it and scan the next one. To guarantee a high system throughput in CRNs, the channel state of PUs needs to be accurately detected to reduce conflict. Sensing optimal idle channel for future transmission is the hot research topic in CRN environment. Probability of available free channel will be less when arrival rate of primary user to their allocated licensed band is high. So the effective channel selection and switching strategy needed to overcome this issue and increasing throughput of the data transmission over cognitive radio network. A
CR system has the ability to adjust its operating parameters, observe the results and eventually take actions and to move the radio toward some optimized operational state. In such a process, learning mechanisms that are capable of exploiting measurements sensed from the environment, gathered experience and stored knowledge are judged as rather beneficial for guiding decisions and actions.

CR combines sensing, learning, and optimization algorithms to control and adapt the radio system from the physical layer and up the communication stack. To this effect, many different learning techniques are available and can be used by a CR, ranging from pure lookup tables to arbitrary combinations of soft computing techniques, which include among others: Artificial Neural Networks, evolutionary/Genetic Algorithms, reinforcement learning, fuzzy systems, Hidden Markov Models (HMMs) etc. The proposed work contributes in this direction, aiming to develop a learning scheme and work towards solving problems related to learning phase of CR systems. In the near future, such learning schemes are expected to assist a CR system to compare among the whole of available, candidate radio configurations and finally select the best one to operate in. Learning Models are also built towards finding parameters to decide which the best configuration to operate with is $[6,7]$. Some learning models use supervised algorithms while certain use unsupervised algorithms such as self organized maps [8].

In the present paper a Neural network training algorithm for spectrum sensing in CRNs is proposed, which employs L-M algorithm. The proposed scheme is used for prediction and to train and test the network. The performance of the present L-M algorithm is compared with HMM and random methods. Numerical results show that the proposed approach for prediction of channel state is effective. The objective of the present work is to evaluate the accuracy of the L-M method using extensive simulations. 
The rest of the paper is organized as follows: section II considered by the authors were the standard, the batch, and provides the brief review on related works, section III the resilient back propagation algorithm. Nouir et al. [22] illustrates our proposal of the system model using the L-M compared the performance of the standard back algorithm and section IV analyses the model's propagation with and Levenberg-Marquardt algorithms to performance using computer simulations. Finally, a brief the prediction of a radio network planning tool. conclusion is made in section $\mathrm{V}$.

\section{RELATED WORK}

In order to assign a channel in $\mathrm{CRN}$ different issues of spectrum availability has to be considered. Based on observing local interference patterns [9] various distributed approximations and maximizing system utility [10] with co ordinations between different CR nodes were proposed. Yuan et.al. [11] proposed a time spectrum model of the available band. Several efforts have been recorded in the literature that aim at combining artificial intelligence and machine learning techniques with cognitive radio technology.

Multilayer Back Propagation Neural Network [12] is used for training in different applications. Multilayered Feedback Neural Network (MFNN) [13] is used as an effective technique for real-time characterization of the communication performance and therefore offers some interesting learning capabilities. A distributed cognitive network access scheme is presented in [14], with the objective to provide the best Quality of Service (QoS), with respect to both radio link and core network performance and user application requirements, by using Fuzzy Logic-based techniques.

Integration of SUs to improve the efficiency of spectrum access and allow the relaxation of constraints at individual SUs is discussed $[15,16]$. Optimal multi-channel cooperative sensing algorithms [17], are considered to maximize the SU throughput subject to per channel detection probability constraints. Mokhnache and Boubakeur [18] compared the performance of three backpropagation algorithms, Levenberg-Marquardt, back propagation with momentum and back propagation with momentum and adaptive learning rate to classify the transformer oil dielectric and cooling state. Kisi and Uncuoglu [19] compared Levenberg-Marquardt, conjugate gradient and resilient algorithm for stream-flow forecasting and determination of lateral stress in cohesion less soils. They found that Levenberg-Marquardt algorithm was faster and achieved better performance than the other algorithms in training. Esugasini et al. [20] compared the classification accuracy of the standard steepest descent back-propagation algorithm against the classification accuracy of the gradient descent with momentum and adaptive learning, resilient back propagation, Quasi-Newton and Levenberg-Marquardt algorithm. The simulations show that the neural network using the Levenberg-Marquardt algorithm achieved the best classification performance.

Iftikhar et al. [21] employed three neural networks with different algorithms to the problem of intrusion detection in computer and network systems. The learning algorithms

\section{III.Proposed SySTEM MODEL}

\section{A. Artificial Neural Network - A Brief Review}

An artificial neural network (ANN) is an effective data modeling scheme used to model and learn both linear and non-linear input/output interactions by training. ANN [23] is a powerful tool to analyze, model and predict. A neural network (NN) is a massively parallel distributed processor made up of simple processing units, which has a natural propensity for storing experimental knowledge and making it available for use [24]. A neural network consists of a pool of simple processing units called 'neurons'. ANNs are made up of artificial neurons interconnected with each other to form programming structures that process the biological neurons by self organizing and learning. A neural network consists of three layers named as input layer, hidden layer and the output layer.

The neural network will work in two phases, one is the training phase and the other is the testing phase. Depending on the pattern of connections a NN can be classified into two basic categories. (a) Feed-forward NNs, where data enters at the inputs and passes through each layer of the network to obtain the outputs. (b) Recurrent NNs, that contain feedback connections, extending from outputs of neurons to inputs within the same or previous layers and vice versa. According to the application, a NN has to be configured such that the application of a set of inputs produces the desired set of outputs which can be achieved by properly adjusting the weights ' $w$ ' among all neuron pairs. The propagation is typically tempered by a constant or bias value, $b$. This bias value is controlled by the user, so that a known input vector may produce the desired output value. This process is called learning or training. Learning can be categorized as supervised and unsupervised learning. In supervised learning, the NN is fed with teaching patterns and trained by allowing it to change its weights according to some learning rule. In unsupervised learning the NN discovers features of the input data in a statistical manner by developing its own ways of classifying the input irritants. Figure 1 shows a three layer feed-forward Artificial neural network

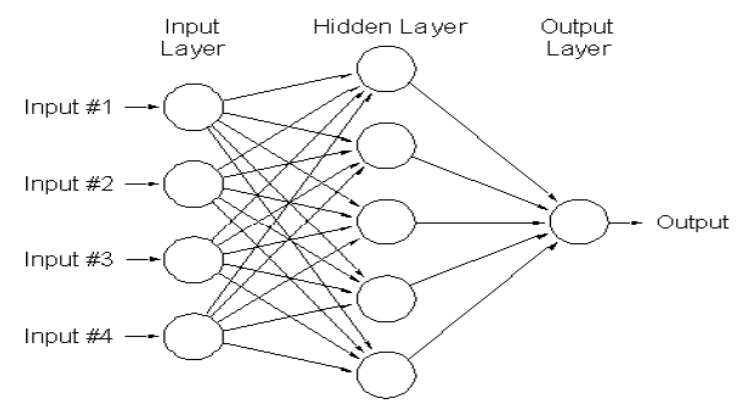

Fig. 1 Artificial neural network 


\section{B. Levenberg-Marquardt Algorithm}

The Levenberg-Marquardt algorithm [25, 26], The simulations are carried out in MATLAB to verify the independently developed by Kenneth Levenberg and performance of the prediction model by Artificial Donald Marquardt. The LM algorithm is an iterative Intelligence (AI) based Neural networks through L-M technique that locates the minimum of a multivariate function that is expressed as the sum of squares of nonlinear real valued functions. It is fast and has stable convergence. In the artificial neural-networks field, this algorithm is used for training networks. The LM algorithm can be thought of as a combination of the steepest descent method and the Gauss-Newton algorithm which inherits the speed advantage of the Gauss-Newton algorithm and the stability of the steepest descent method.

Levenberg-Marquardt algorithm [27] is considered an approximation to Newton's method. Let the function to be minimized be denoted as $F n(v)$ and it is minimized with respect to the parameter vector denoted as $v$. The technique can be given by:

$$
\Delta v=-\left[\nabla^{2} F n(v)\right]^{-1} \nabla F n(v)
$$

Here, $\nabla^{2} F n(v)$ is the Hessian matrix and $\nabla F(a)$ is the gradient matrix. The gradient matrix is taken as the sum of squares of the error function given by:

$$
F n(v)=\sum_{i=1}^{N_{c}} e_{i}^{2}(v)
$$

Here, it can be given that:

$$
\nabla^{2} F n(v)=J^{T}(v) J(v)+D(v)
$$

Where, $J()$ is the Jacobian matrix, and

$$
\begin{aligned}
& D(v)=\sum_{i=1}^{N_{c}} e_{i}(v) \nabla^{2} e_{i}(v) \\
& \Delta v=\left[J^{T}(v) J(v)+\mu I\right]^{-1}+J^{T}(v) e(v)
\end{aligned}
$$

Initially, all the inputs are given to the network and corresponding network outputs are calculated. Then the sum of squares of errors over all inputs $F n(v)$ and the Jacobian matrix ' $J$ 'is calculated to solve $\Delta v$. The sum of squares of errors is recalculated using $v+\Delta v$.If this new sum of squares is less than that earlier computed, then ' $\mu$ ' is reduced by ' $\beta$ ' and updated $\nu=v+\Delta v$ and go back to initial step. Here ' $\beta$ ' is the learning rate and ' $\mu$ ' is the step size. If the sum of squares is not decreased, then increase ' $\mu$ ' by ' $\beta$ ', and go back to step to solve for $\Delta v$. The algorithm is assumed to have converged when the normal of the gradient is less than some predetermined value, or when the sum of squares has been lessened to error goal. The key step in this algorithm is the computation of the Jacobian matrix. For the neural network problem the terms in the Jacobian matrix can be computed by a simple modification to the back propagation algorithm.

Copyright to IJARCCE algorithm. Figures 2-4 illustrates throughput graphs for increasing channel count. It is observed that the throughput performance increases with the increase in time slots and also with increase in number of channels. The extensive simulated results show that, such a solution is better than HMM [28]. The proposed channel prediction model can be used to detect the spectrum hole in CRN. The maximum throughput value obtained represents the superior performance of L-M algorithm. The statistics of throughput performance analysis by the three, five and seven channel count is summarized. Fig 2 shows the percentage of time during which the SU can successfully transmit the data without colliding with the PU in a CRN for three channel state, where as the fig 3 and 4 shows for the five and seven channels respectively. Overall, in this paper we demonstrated efficiency of L-M algorithm over statistical spectrum allocation techniques in the context of throughput for time varying channels in CRNs.

\section{Conclusions}

In this paper, Artificial Intelligence based neural network L-M algorithm is implemented to solve current wireless network problems for cognitive radios, resulting from the limited available spectrum for throughput maximization. The estimates shows the performance and efficiency of the LM method when the channel count changes monotonically. In the present work, the experiments for the performance of spectrum sensing in cognitive radio networks are carried out for time slots zero to100. Proposed L-M based neural network gives better output when compared with currently available statistical learning techniques such as HMM and random techniques. Numerical results show that it remarkably improves the throughput performance. Additionally HMM is not much good in prediction compared with the AI based prediction schemes. In order to achieve better spectrum sensing and to overcome the limitations of the proposed technique for channel status in cognitive radio, optimization procedures can be attempted by hybridizing L-M based neural network with recently available algorithms.

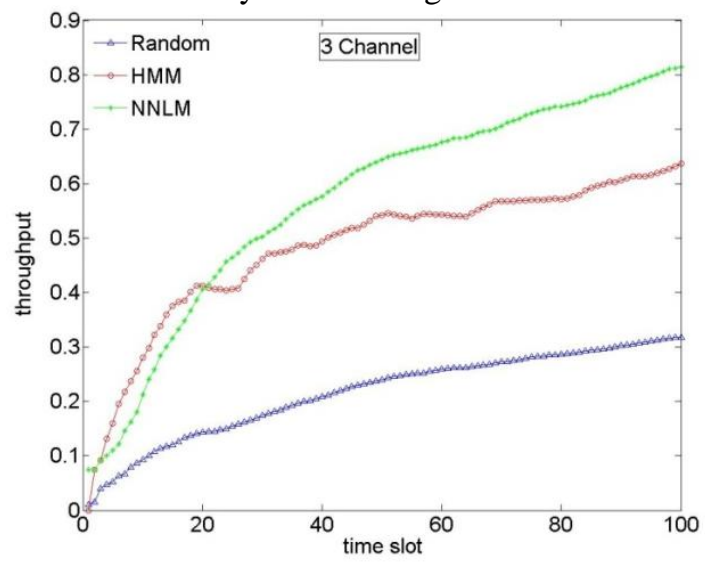

Fig. 2. Throughput plot for three channel state 


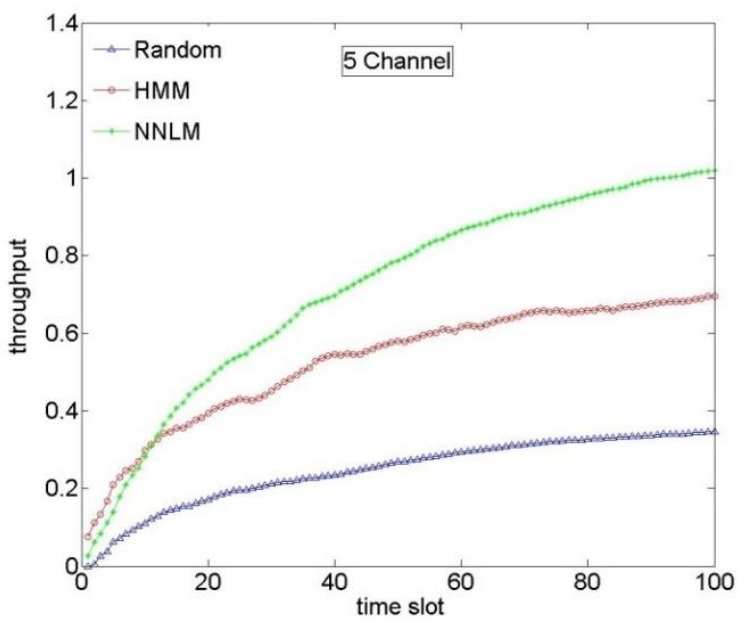

Fig. 3. Throughput plot for five channel state

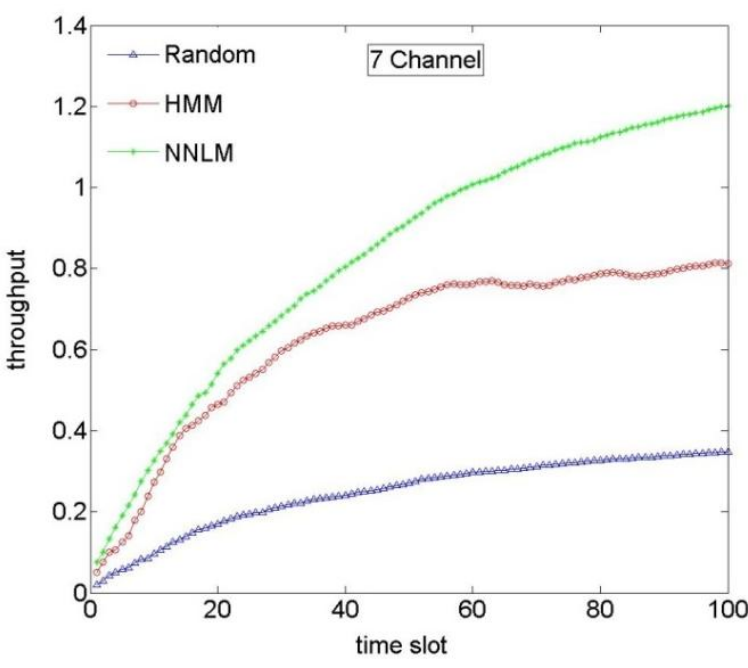

Fig. 4. Throughput plot for seven channel state

\section{REFERENCES}

[1] Federal Communication Commission: 'Spectrum policy task force report, FCC 02-155, November 2002

[2] Federal Communications Commission. Notice of proposed rulemaking, in the matter of unlicensed operation in the TV broadcast bands (docket no. 04-186) and additional spectrum for unlicensed devices below $900 \mathrm{MHZ}$ and in the $3 \mathrm{GHZ}$ band (02380), FCC 04-113. May 2004

[3] http://grouper.ieee.org/groups/802/22/. IEEE 802.22, Working Group on Wireless Regional Area Networks (WRAN).

[4] J. Mitola and G. Q. Maguire, "Cognitive radios: Making software radios more personal,” IEEE Pers. Commun., vol. 6, no. 4, pp. 13 18, Aug. 1999.

[5] J. Mitola, "Cognitive radio: An integrated agent architecture for software defined radio,” PhD. diss., Royal Inst. Technol. (KTH), Stockholm, Sweden, 2000

[6] K. Tsangaris, A. Katidiotis, P. Demestichas, "Neural networkbased learning schemes for cognitive radio systems", International Journal on Computer Communication, (2008) 3394-3404

[7] K. Tsangaris, A. Katidiotis, P. Demestichas, "Performance evaluation of artificial neural network-based learning schemes for cognitive radio systems", International Journal on Computers and Electrical Engineering (2010) 518-535,

[8] Kostas Tsagkaris , Aimilia Bantouna, Panagiotis Demestichas, "Self-Organizing Maps for advanced learning in cognitive radio systems", Elsevier, International Journal on Computers and Electrical Engineering, Article in Press 2012

[9] H. Zheng and L. Cao, "Device-centric spectrum management," in Proc. DySPAN, 2005

Copyright to IJARCCE
[10] L. Cao and H. Zheng., "On the efficiency and complexity of distributed spectrum allocation," in Proc. of Crown Com, 2007.

[11] Y. Yuan, P. Bahl, R. Chandra, T. Moscibroda, and Y. Wu, "Allocating dynamic time-spectrum blocks in cognitive radio networks," in Proc. Of ACM MobiHoc, 2007, pp. 130 - 139.

[12] Z. Zhang and X. Xie, "Application research of evolution in cognitive radio based on GA," 3rd IEEE Conference on Industrial Electronics and Applications (ICIEA 2008), 2008.

[13] N.Baldo and M. Zorzi, "Learning and Adaptation in Cognitive Radios Using Neural Networks", 5th IEEE Consumer Communications and Networking Conference (CCNC 2008), 2008.

[14] M. Baldo, M. Zorzi, "Cognitive Network Access using Fuzzy Decision Making," in Proc. of the 1st IEEE CogNet 2007 Workshop (in conjunction with IEEE ICC 2007), Glasgow, UK, June 2007 Communications, 2007. ICC '07. IEEE International Conference on , vol., no., pp.6504-6510, 24-28 June 2007.

[15] T. Do and B.L. Mark. "Joint spatial-temporal spectrum sensing for cognitive radio networks". In proc. of CISS, pages 124-129, march 2009

[16] E. Visotsky, S. Kuffner, and R. Peterson, "On collaborative detection of TV transmissions in support of dynamic spectrum sharing”, In proc. Of DySPAN, pages 338 -345, nov. 2005.

[17] R. Fan and H. Jiang, "Optimal multi-channel cooperative sensing in cognitive radio networks", IEEE Transactions on Wireless Communications, 9(3):1128-1138, 2010.

[18] Mokbnache L, Boubakeur, "A Comparison of Different BackPropagation Algorithms used in The Diagnosis of Transformer Oil”, IEEE Annual Report Conference on Electrical Insulation and Dielectric Phenomena: 244-247, 2002.

[19] Kisi O, Uncuoglu E "Comparison of Three Back-Propagation Training Algorithms for Two Cases Studies", Indian Journal of Engineering \& Materials Sciences. 12: 434-442, 2005.

[20] Esugasini E, Mashor MY, Isa N, Othman N, "Performance Comparison for MLP Networks Using Various Back Propagation Algorithms for Breast Cancer Diagnosis" Lecture Notes in Computer Science. 3682 : 123-130, 2005.

[21] Iftikhar A, Ansari MA, Mohsin S, " Performance Comparison between Back propagation Algorithms Applied to Intrusion Detection in Computer Network Systems", 9th WSEAS International Conference on Neural Networks (NN'08): 231-236. 2008.

[22] Nouir Z, Sayrac ZB, Fourestie B, Tabbara W, Brouaye F "Comparison of Neural Network Learning Algorithms for Prediction Enhancement of a Planning Tool", http://confs.comelec.telecomparistech.fr/EW2007/papers/1569014736.pdf, 2008.

[23] Martin T. Hagan and Mohammad B. Menhaj, "Training Feed forward Networks with the Marquardt Algorithm", IEEE Transactions On Neural Networks, Vol. 5, No. 6, Pp.989-994, 1994.

[24] Simon Haykin, "Neural Networks: A Comprehensive Foundation", 2nd ed. South Asia: Pearson Education and Dorling Kindersley Pvt.Ltd, 1999.

[25] K. Levenberg, " A method for the solution of certain problems in least squares, Quarterly of Applied Mathematics", 5, 164-168, 1944.

[26] D. Marquardt, "An algorithm for least-squares estimation of nonlinear parameters", SIAM Journal on Applied Mathematics, 11(2), 431-441, June 1963.

[27] D. Marquardt, "An algorithm for least squares estimation of nonlinear parameters,” J. Soc. Ind. Appl. Math., pp. 43141, 1963.

[28] Pavithra Roy P., and M. Muralidhar., "Hidden Markov Model based Channel state prediction in cognitive radio networks", International Journal of Engineering Research \& Technology, Vol. 4 Issue 02 , February-2015.

\section{BIOGRAPHIES}

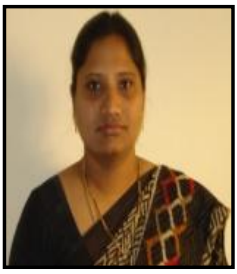

Pavithra Roy P. obtained her Bachelor's degree in Electronics and Communication Engineering from Jawaharlal Nehru Technological University, Hyderabad, AP, India. Then she obtained her Master's degree in Electronics and Communication Engineering with specialization in Digital Electronics and Communication Systems from the Jawaharlal Nehru 
Technological University, Anantapur, AP, India. Currently, she is an Assistant Professor at the Faculty of Electronics and Communication Engineering, Vemana Institute of Technology, Bangalore, India. Her specializations include Communication Systems, Logic Design and Neural Networks. Her current research interests are Wireless Communications, Cognitive Radio Systems, and Spectrum Utilization.

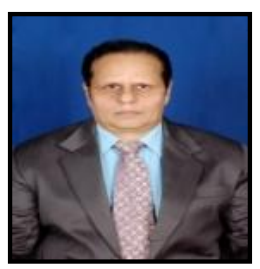

Dr. Muralidhar M. (Muralidhar Mahankali.) received B.E., M.E. and Ph.D degrees from Sri Venkateswara University, Tirupati, Andhra Pradesh, India. He worked in different cadres in the department of Electrical \& Electronics Engineering in Sri Venkateswara University, Tirupati, Andhra Pradesh, India from 1977 to 2011. Currently he is working as Principal and Professor in EEE in Sri Venkateswara College of Engineering \& Technology (Autonomous), Chittoor, Andhra Pradesh, India. His research interests are Wireless sensor networks and heterogeneous wireless networks. He is a senior Member of the Institution of Engineers (India) and a Member of the Chartered Engineers (India). 